\title{
Drosophila motor axons recognize and follow a Sidestep-labeled substrate pathway to reach their target fields
}

\author{
Matthias Siebert, Daniel Banovic, Bernd Goellner, and Hermann Aberle ${ }^{1}$ \\ Westfälische Wilhelms-Universität Münster, Institut für Neurobiologie, 48149 Münster, Germany
}

\begin{abstract}
During development of the Drosophila nervous system, migrating motor axons contact and interact with different cell types before reaching their peripheral muscle fields. The axonal attractant Sidestep (Side) is expressed in most of these intermediate targets. Here, we show that motor axons recognize and follow Side-expressing cell surfaces from the ventral nerve cord to their target region. Contact of motor axons with Side-expressing cells induces the down-regulation of Side. In the absence of Side, the interaction with intermediate targets is lost. Misexpression of Side in side mutants strongly attracts motor axons to ectopic sites. We provide evidence that, on motor axons, Beaten path Ia (Beat) functions as a receptor or part of a receptor complex for Side. In beat mutants, motor axons no longer recognize Side-expressing cell surfaces. Furthermore, Beat interacts with Side both genetically and biochemically. These results suggest that the tracing of Side-labeled cell surfaces by Beat-expressing growth cones is a major principle of motor axon guidance in Drosophila.
\end{abstract}

[Keywords: Drosophila; motor axon guidance; Sidestep; Beaten path; substrate pathway; in vivo imaging; time-lapse movie]

Supplemental material is available at http://www.genesdev.org.

Received January 5, 2009; revised version accepted March 18, 2009.

Wiring of the nervous system is a precisely controlled process that includes axon outgrowth, axon pathfinding, target recognition, and synapse formation. Outgrowing axons of the same subtype often join and migrate collectively toward their targets, a process called selective fasciculation. Upon arrival in the target region, a subset of growth cones must inevitably defasciculate from the main pathway to select a specific synaptic target. Advancing growth cones are believed to express appropriate receptors that detect and evaluate relevant guidance molecules presented by surrounding cells and tissues. Despite the discovery of several conserved regulators of axonal pathfinding, the spatiotemporal sequence of molecular events that steer growth cones is still unclear (Dickson 2002; Araujo and Tear 2003; Huber et al. 2003). How exactly do growth cones recognize and interpret guidance cues to make pathway decisions? Over the years, several models have been proposed to describe general features of axon navigation across species, including the chemoaffinity hypothesis (Sperry 1963), the guidepost cell hypothesis (Bate 1976), the blueprint hypothesis (Singer et al. 1979), and the labeled pathways hypothesis (Goodman et al. 1983).

${ }^{1}$ Corresponding author.

E-MAIL aberleh@uni-muenster.de; FAX 49-251-8324686.

Article published online ahead of print. Article and publication date are online at http://www.genesdev.org/cgi/doi/10.1101/gad.520509.
The labeled pathways hypothesis postulates that a small number of early differentiating neurons pioneer a stereotypic array of differentially labeled axonal pathways (Goodman et al. 1983; Raper et al. 1983b). These primary pathways are selectively recognized by subsequently developing growth cones (Raper et al. 1983a). While follower axons migrate along a homogenous substrate toward their target region, pioneer axons have to interact with many different substrates, actively search for guidance cues, and interpret relevant guidance information. Pathfinding decisions for pioneer axons are greatly simplified if directional cues would be present in the substrate. However, the experimental evidence for the existence of such substrate pathways is scarce. Ghysen and Janson (Ghysen 1978; Ghysen and Janson 1980) noted that projections of sensory axons in homeotic mutants of Drosophila involved the specific recognition of "pre-existing trails." Similarly, axons emanating from transplanted eyes or Mauthner neurons in Xenopus have been observed to project along defined tracks called "substrate pathways" (Katz and Lasek 1979, 1981). Furthermore, Silver and Rutishauser (1984) reported that chick optic axons are guided along a preformed adhesive pathway. Despite the appealing simplicity of substrate pathways, the molecular tags that provide directional information have not been identified.

One possible molecule that could serve as a directional cue for Drosophila motor axons is the attractant Sidestep 
(Side), a transmembrane protein of the immunoglobulin superfamily that is dynamically expressed during embryogenesis but prominently enriched in muscles when motor axons arrive in their target areas (Sink et al. 2001). In side mutant embryos, motor axons fail to defasciculate at key choice points and hence bypass their muscle targets, suggesting that Side functions as a target-derived attractant (Sink et al. 2001; de Jong et al. 2005). Interestingly, mutations in beaten path Ia (beat) lead to similar axon guidance phenotypes, and like Side, Beat has been shown to regulate axon defasciculation at choice points (Fambrough and Goodman 1996; Holmes and Heilig 1999; Sink et al. 2001). Based on primary structure predictions, Beat is a secreted protein of the immunoglobulin superfamily that has been shown to function as an anti-adhesive factor on motor axons (Fambrough and Goodman 1996; Pipes et al. 2001). Beat consists of two Ig domains and a Cysteine-rich C-terminal domain that shares similarities with cystine knots (Bazan and Goodman 1997; Mushegian 1997).

Here, we show that side encodes a candidate directional cue that steers Drosophila motor axons from the place of their birth to their peripheral targets. The spatiotemporal expression pattern of Side thus delineates a linear pathway for motor axons. High levels of Side are consistently found ahead of Beat-expressing growth cones. Contact with motor axons induces the downregulation of Side and eliminates the pathway, preventing other outgrowing nerves from choosing the same route. Furthermore, we provide biochemical and genetic evidence that Beat functions as a membrane-associated receptor or part of a receptor complex for Side. Taken together, these findings support the concept of preexisting pathways labeled with molecular markers that are recognized by a specialized subset of axons expressing the appropriate receptors.

\section{Results}

Neuromuscular connectivity in Drosophila is established by efferent motor axons that project in a stereotypical pattern from the ventral nerve cord to peripheral body wall muscles (Sink and Whitington 1991; van Vactor et al. 1993). To visualize this dynamic process in living embryos, we examined exon trap lines in the fasciclin II (fasII) locus that specifically label motor axons with green fluorescent protein (GFP) (Rasse et al. 2005; Buszczak et al. 2007; Stork et al. 2008). We decided to use the homozygous viable line FasIIGFP ${ }^{\text {Mue397 }}$ that contains an insertion in the extracellular domain of FasII (Rasse et al. 2005; Stork et al. 2008). FasIIGFP ${ }^{\text {Mue397 }}$ stains all tissues known to express endogenous FasII, as recognized by the monoclonal anti-FasII antibody 1D4 (Grenningloh et al. 1991). However, GFP was also detectable in the hemolymph of FasIIGFP ${ }^{\text {Mue397 }}$ embryos (Supplemental Fig. S1). Since FasIIGFP ${ }^{\mathrm{Mue} 397}$ is expressed in motor neurons prior to axogenesis, it is an excellent marker for early developmental stages.

\section{Motor axons follow a Sidestep-labeled pathway}

The axon guidance molecule Sidestep (Side) attracts motor axons and is expressed in a dynamic pattern in neurons and muscles during embryogenesis (Sink et al. 2001), prompting us to examine its spatiotemporal relationship to motor axons. At embryonic stage 12, Side was expressed in a belt-like pattern along the ventral midline (Fig. 1A-C). The pioneering motor neuron of the
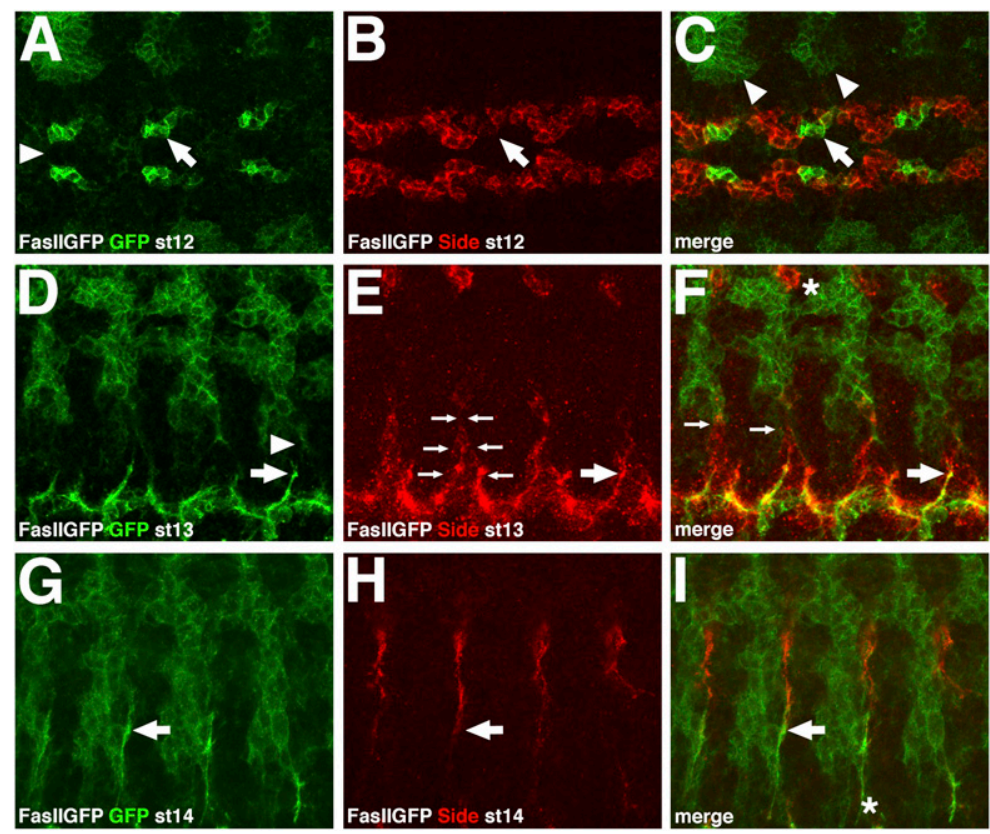

Figure 1. Motor axons follow Sidestep-labeled cell surfaces. $(A-I)$ FasIIGFP $^{\text {Mue } 397}$ embryos stained with anti-GFP and anti-Side antibodies to visualize the spatiotemporal relationship of motor axons and Sideexpressing cells. $(A-C)$ Ventral view of a stage $12 \mathrm{em}$ bryo. (A) FasIIGFP ${ }^{\text {Mue } 397}$ is expressed in a cluster of four to five cells in each neuromere, including the aCC and pCC neurons. Arrowhead marks the ventral midline. $(B)$ Sidestep is expressed in a belt-like pattern flanking the ventral midline. $(C)$ FasIIGFP $^{\text {Mue397 }}$-positive cells are located next to Side-expressing cells (cf. arrows in $A-C)$. Arrowheads mark the tracheal precursors. Anterior is left. $(D-F)$ Ventrolateral view of a stage 13 embryo. ( $D$ ) Pioneering axons of the ISN (arrow) project toward the exit junction and developing ganglionic branch of the trachea (arrowhead). (E) Sidestep is expressed in a triangular area (small arrows) in the CNS that points toward the trachea. $(F)$ Motor axons follow Sidestep-positive cell surfaces toward the trachea (arrows in $D-F$ ). Small arrows in $F$ mark the close proximity of the tip of the triangle to the trachea. Asterisk indicates the earliest expression of Side in sensory neurons of the dorsal cluster. $(G-I)$ Lateral view of a stage 14 embryo. $(G)$ ISN motor axons migrate dorsally between the trachea and the developing muscle field. $(H)$ Sensory axons grow ventrally along similar routes. $(I)$ Sensory axons and motor axons fasciculate in the ventrolateral region of the embryo (arrows in $G-I$ ). The asterisk in $I$ shows that Side is not detectable any more in the triangular area of the CNS (cf. F). 
intersegmental nerve (ISN), the aCC neuron, developed next to Side-expressing cells (Fig. 1A-C, arrows). At stage 13, the Side expression domain had changed into a triangular pattern, with the tips pointing away from the midline (Fig. 1E). Motor axons of the ISN projected along the anterior edge of each triangle (Fig. 1D-F, arrows). Remarkably, axons of the segmental nerve (SN) grew along the posterior edge. The triangular cell cluster guided motor axons directly toward the exit junction and the incoming ganglionic branch of the trachea (Fig. $1 F$, small arrows). At the trachea, motor axons fasciculated with Side-positive, afferent sensory axons exactly at the lateral bidendritic neuron (lbd) (Fig. 1G-I, arrows). When the ISN reached the end of the sensory tracks at the dorsal bidendritic neuron (dbd), expression of Side in sensory neurons was no longer detectable but now could be observed in muscle fibers (Sink et al. 2001). Side-expressing muscles likely attract motor axons, causing them to leave the sensory tracks. In general, cells and tissues located ahead of the growth cones express Side. Upon contact with motor axons, the expression of Side is down-regulated in the substrate, as monitored with antiSide antibodies (Fig. 1I, asterisk) and in situ hybridizations (Sink et al. 2001). The spatiotemporal expression pattern of Side is therefore consistent with a cell surface marker that prefigures and potentially directs the path of motor axons.

Sensory tracks cannot be recognized in side mutants

If motor axons are attracted to and migrate along Sidestep-expressing sensory axons, then this interaction should be disrupted in side mutants. We examined the fasciculation of motor and sensory axons in FasIIGFP $^{\text {Mue397 }}$ embryos stained with anti-Futsch antibodies that recognize a microtubule-associated protein (Hummel et al. 2000). In wild-type embryos, ISN motor axons remained tightly fasciculated with sensory axons until they reached the dbd neuron (Fig. 2A). Only 2\% of ISN nerves showed detachments $>4 \mu \mathrm{m}$ (Table 1). In contrast, in side mutants, $49 \%$ of ISN motor nerves were detached from sensory nerves of the anterior fascicle (Fig. 2B; Table 1). This phenotype was even stronger when Side was overexpressed in muscles using the musclespecific driver Mef2-Gal4 (Fig. 2C). In these embryos, $84 \%$ of motor nerves were detached (Table 1). These results show that the mutual recognition of motor and sensory axons is disturbed when levels of Side are altered. Side thus functions in the attraction of motor axons to sensory axons. The mutual recognition provides a stationary substrate for the migration of either nerve, and eventually results in the formation of coherent nerve bundles.

\section{Growth cones in side mutants actively search for pathway information}

If motor axons use sensory tracks as growth substrates the absence of Side might affect the rate of growth cone progression. We recorded time-lapse movies of the migration of the ISN in wild-type embryos expressing FasIIGFP $^{\text {Mue397 }}$ and compared them with side mutants (see the Materials and Methods). In wild-type embryos, the ISN emerged as a thin bundle of axons in lateral body wall regions at early stage 14 (Fig. 2D). The growth cone advanced continuously until it reached the dorsal trunk,

Figure 2. The ISN detaches from sensory axons and shows migratory delays in side mutants. $(A-C)$ Motor axons are stained with anti-GFP and sensory axons with anti-Futsch antibodies. (A) In stage 15 wild-type embryos, the ISN is tightly associated with sensory axons of the anterior fascicle (arrows) until it reaches the dbd neuron (arrowhead). The dbd neuron stains only weakly with anti-Futsch antibodies at this stage, as do motor axons. $(B)$ The ISN is partially detached from sensory axons (arrows) prior to the dbd (arrowhead) in side ${ }^{\mathrm{C} 137}$ / side ${ }^{\text {I1563 }}$ mutant embryos. $(C)$ Wild-type embryos overexpressing Side in muscles using Mef2-Gal4 show severe detachments of motor and sensory axons (arrows). For quantification of the detachment phenotypes see Table 1. $(D-F)$ Stills from Supplemental Movie 2-1 showing the migration of the ISN in the lateral body wall of a FasIIGFP ${ }^{\text {Mue397 }}$ embryo at stage 14 . The growth cone of the ISN (arrows) travels between the transverse connective (downward arrowheads) and the myogenic field (asterisks). The growth cone marked with arrows makes a small detour but corrects its path. Time is indicated in hours and minutes (h:min). (G-I) Stills from Supplemental Movie 2-2 showing the advance
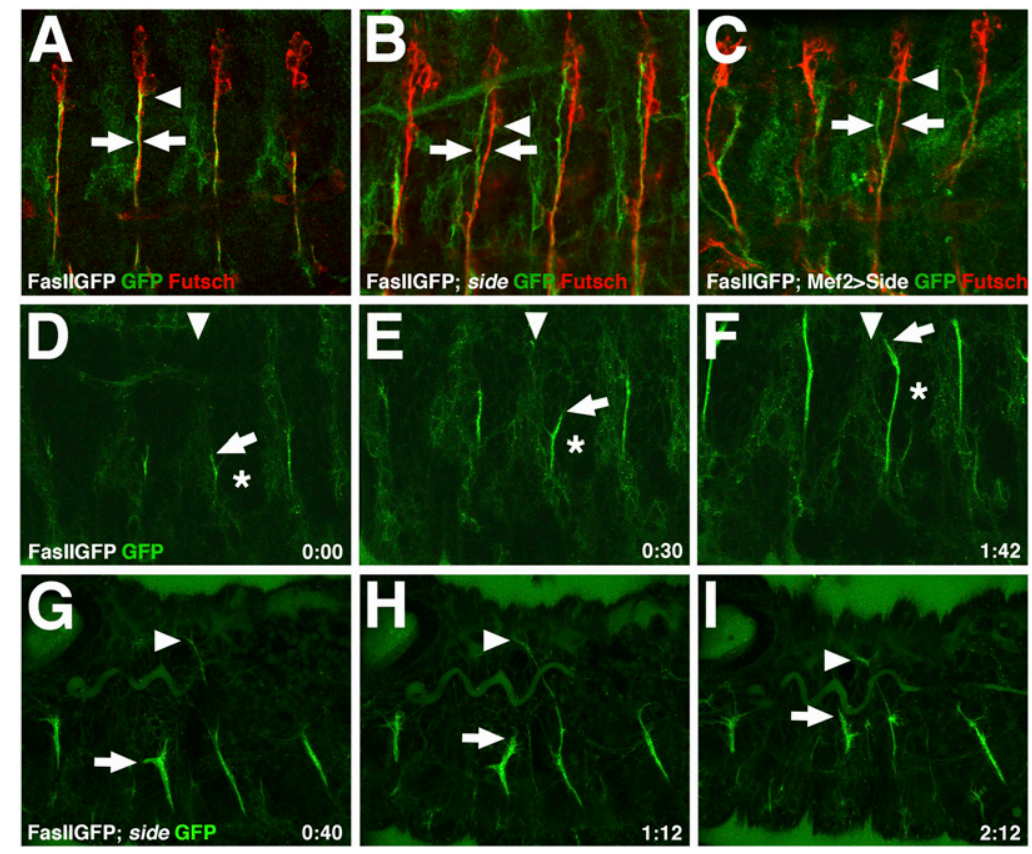

of the ISN in a side $\mathrm{C}^{\mathrm{C} 37} / \mathrm{side}^{\mathrm{Il} 563}$ mutant embryo at

stage 16. While one of the ISN nerves has already crossed the dorsal trunk (arrowheads), others are lacking behind (arrows). Delayed growth cones are larger and sprout more filopodia. 
Table 1. Frequencies of detachments of ISN motor axons and sensory axons of the anterior fascicle between the lbd and dbd

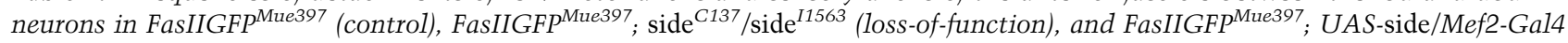
(gain-of-function) embryos

\begin{tabular}{|c|c|c|c|}
\hline Detachments of the ISN/anterior fascicle & FasIIGFP ${ }^{M u e 397}$ & $\begin{array}{l}\text { FasIIGFP }^{\text {Mue397 }} ; \\
\text { side }^{\mathrm{C} 137} / \text { side }^{\mathrm{I1} 563}\end{array}$ & $\begin{array}{c}\text { FasIIGFP }^{\text {Mue397 }} ; \\
\text { Mef2-Ga14/UAS-side }\end{array}$ \\
\hline Percent detachments & $2 \%$ & $49 \%$ & $84 \%$ \\
\hline$n$ hemisegments & 62 & 67 & 45 \\
\hline
\end{tabular}

Only detachments $>4 \mu \mathrm{m}$ in stage 17 embryos have been evaluated.

with an average growth rate of $31.9 \pm 13.4 \mu \mathrm{m} / \mathrm{h}(\mathrm{n}=16)$. The entire fascicle employed only a single, relatively small growth cone for steering, which increased in brightness and thickness over time (see Supplemental Movie 2-1). It is interesting to note that during its migration along the transverse branch of the trachea, the ISN was not attracted into the nearby myogenic field (Fig. 2D-F, asterisks). The growth cones occasionally diverged from their normal paths, which caused migratory delays but no permanent guidance defects, suggesting that growth cones have the inherent ability to correct minor misprojections (Fig. 2D-F, arrows). In side mutant embryos, we observed severe delays in the dorsal migration of the ISN (see Supplemental Movie 2-2). Growth cones eventually managed to cross the dorsal trunk (Fig. 2G-I, arrowheads), but a subset lagged behind (Fig. 2G-I, arrows). The growth rates were strongly reduced compared with wild-type nerves $(15.2 \pm 4.6 \mu \mathrm{m} / \mathrm{h} ; n=12)$. Stalled growth cones appeared thicker and sprouted more and longer filopodia. A small percentage of growth cones exhibited highly disoriented directionality, extending in abnormal directions over the course of axonal growth (Fig. 2G-I, cf. arrows). Taken together, the reduced growth rates and the disoriented pattern of growth cone extensions indicate that motor axons in side mutants increase their search behavior, likely due to the absence of attractive cues in the substrate.

\section{Side instructs the growth direction of motor axons}

If Side marks a path for motor axons it should be possible to redirect the growth direction of motor axons by creating an ectopic pathway. Misexpression of Side on trachea or a subset of muscles in wild-type embryos results in extended contact and exploration by motor nerves (Sink et al. 2001). To circumvent the presence of endogenous Side we expressed Side in the trachea of side mutants using breathless-Gal4. Under these conditions, most, if not all, ISN growth cones were tightly attached to tracheal branches (Fig. 3B, arrows). Even more strikingly, the ISN followed the tracheal substrate and crossed and recrossed the transverse connective. In comparison, in side mutant control embryos, the ISN grew out into the periphery but none of the growth cones was particularly associated with tracheal branches (arrows in Fig. 3A). In a second experiment, we expressed Side in highly motile hemocytes. Normally, these circulating cells function in the immune system of Drosophila and do not establish firm contacts with growth cones neither in wild-type nor in side mutant embryos (Fig. 3C). Strikingly, when Side was ectopically expressed in hemocytes of side mutants using Serpent-Gal4, most, if not all, growth cones were attached to hemocytes (Fig. 3D). Filopodial extensions of both cell membranes were tightly interdigitated. Timelapse movies in a FasIIGFP ${ }^{\text {Mue } 397}$ background showed strong mutual adhesion between hemocytes and growth cones (Fig. 3E-H; Supplemental Movie 3-1). In a third experiment, we expressed Side in muscles of wild-type embryos under control of Mef2-Gal4. This manipulation results in the up-regulation of Side when muscles are not yet developed but motor axons are just passing by. Under this condition, the ISN nerves were strongly attracted to the developing muscle field and split aberrantly into several directions, suggesting directed growth toward individual muscle precursors (Fig. 3I,J). The split growth cones prevented dorsal migration, causing strongly reduced growth rates (see Supplemental Movie 3-2). In summary, the ability to redirect the path of motor axons shows that Side is an instructive cue for motor axons independent of the tissue in which it is expressed.

Beaten path Ia (Beat) functions in the recognition of Side-labeled cell surfaces

Since mutations in beaten path Ia (beat) and side have been reported to cause similar motor axon guidance defects, we wondered whether Beat and Side cooperate in pathfinding decisions (Fambrough and Goodman 1996; Sink et al. 2001). To test this possibility, we examined the locations of neuromuscular junctions (NMJs) in beat and side mutant third instar larvae that reflect the final outcome of embryonic projection errors at a high spatial resolution (de Jong et al. 2005). Guidance errors lead to the irreversible formation of NMJs at aberrant positions that can be visualized with the post-synaptic marker CD8-GFP-Sh (Meyer and Aberle 2006). Compared with wild-type larvae (Fig. 4A), NMJs were frequently absent on dorsal muscles $1 / 9$ in both beat and side mutant larvae (Fig. 4B,C; Table 2). In addition, beat and side mutants showed similar innervation defects on a variety of muscles, indicating that they function in a related process.

We next tested for possible activating or antagonizing effects of Beat with Side. We expressed Beat and Side individually or together in muscles of wild-type animals using Mef2-Gal4. Since expression of Side on muscle precursors prematurely attracts motor axons (Fig. 3I,J) we assumed that the simultaneous overexpression of Beat might increase or suppress this effect. While increased levels of Beat had only minor effects on the innervation pattern of dorsal muscles (Fig. 4D), ectopic expression of Side resulted in an almost complete lack of dorsal NMJs 
due to premature attraction into ventral and lateral muscle fields (Fig. 4E; Table 2). Simultaneous expression of Beat and Side in muscles completely abolished the Side gain-of-function phenotype (Fig. 4F; Table 2). Thus, Beat is able to suppress the function of exogenous Side when coexpressed in the same tissue. To collect further evi-
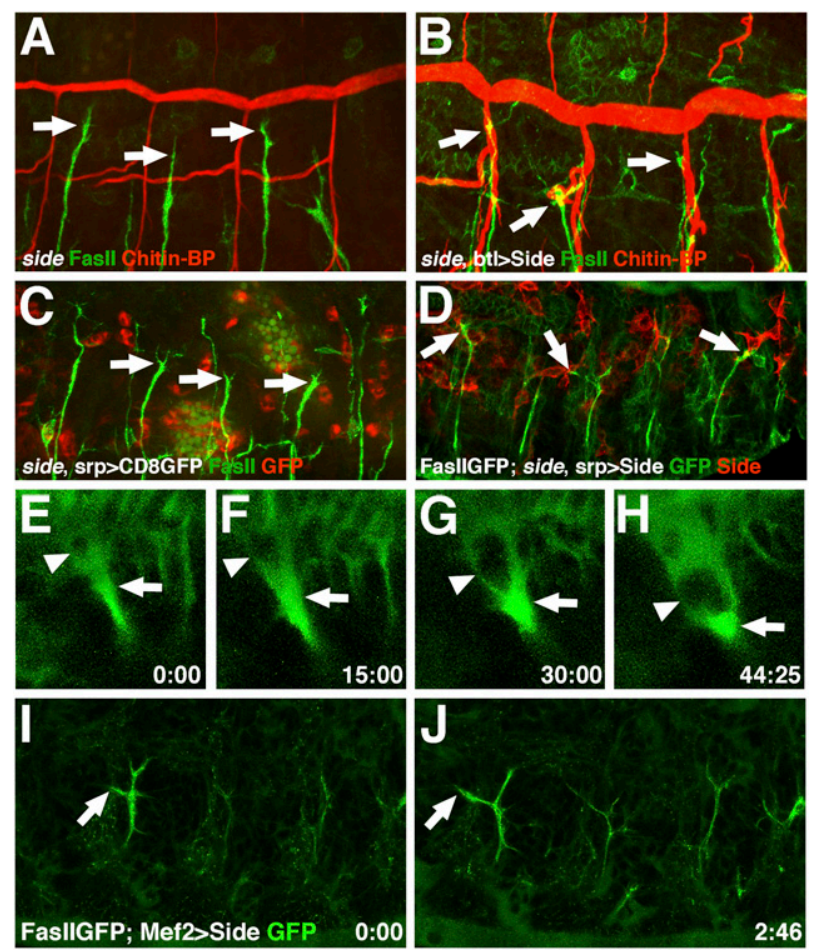

Figure 3. Sidestep is sufficient to direct the path of motor axons. $(A, B)$ Side attracts motor axons to tracheal branches. Axons are stained with anti-FasII antibodies, the tracheal lumen is stained with a chitin-binding probe coupled to Rhodamine. $(A)$ The ISN growth cones are not in direct contact with tracheal branches in a side mutant embryo at stage 16 (arrows) (genotype: side $e^{\mathrm{C} 137} /$ side $\left.^{\mathrm{I1} 1563}\right)$. (B) Ectopic expression of Side in trachea of side mutants strongly attracts motor axons to tracheal branches, the only source of Side in these embryos (arrows) (genotype: btlGal4/+; UAS-side, side ${ }^{\mathrm{C} 137} /$ side $\left.^{\mathrm{I1563}}\right) \cdot(C, D)$ Side attracts motor axons to hemocytes. $(C)$ Stage 16 side mutant embryo expressing transmembrane CD8GFP in hemocytes (red). ISN growth cones misproject but are not in direct contact with hemocytes (arrows) (genotype: UAS-mCD8GFP, side $e^{\mathrm{C} 137} /$ side $^{\mathrm{I1} 563}$, SerpentGal4). (D) FasIIGFP ${ }^{\mathrm{Mue} 397}$ embryo mutant for side and expressing exogenous Side in hemocytes. In the absence of endogenous Side, almost every growth cone of the ISN is in contact with a hemocyte (arrows) (genotype: FasIIGFP ${ }^{\text {Mue397; }}$ UAS-side, side $\mathrm{C}^{\mathrm{C} 137} /$ side $^{\mathrm{I1} 1563}$, Serpent-Gal4). $(E-H)$ Still images of Supplemental Movie 3-1 (time in minutes and seconds) showing strong adhesive interactions between an ISN growth cone (arrow) and a Side-expressing hemocyte (arrowhead) in a side mutant embryo at stage 16 (genotype: FasIIGFP Mue397; UAS-side, side ${ }^{\mathrm{C} 137}$ / side $^{I 1563}$, Serpent-Gal4). $(I, /)$ Stills of Supplemental Movie 3-2 (time in hours and minutes) showing ISN growth cones in a stage 15 FasIIGFP ${ }^{\mathrm{Mue} 397}$ embryo overexpressing Side in muscles (genotype: FasIIGFP ${ }^{\text {Mue397}}$; Mef2-Gal4/UAS-side). The growth cone of the ISN splits into several directions, preventing its dorsal migration and deflecting its route along presumptive muscle fibers (arrows). dence for a genetic interaction, we created double mutants. In beat; side double mutants, the pattern of mislocalized NMJs was qualitatively similar and quantitatively not increased, when compared with single mutants of beat or side (Fig. 4G; Table 2). Since Beat is expressed in motor axons (Fambrough and Goodman 1996), it might function in the detection of Side on substrates. If so, muscles overexpressing Side should not attract motor axons in a beat mutant background, as they normally would in a wild-type background (Figs. 3I,J, 4E). Indeed, overexpression of Side in muscles of beat mutants did not increase the dorsal innervation defects of beat mutants (Fig. 4H; Table 2). Motor axons lacking Beat seem therefore to be unable to respond to Side presented on muscle progenitors, and hence unable to recognize Side-labeled substrates. Genetic evidence thus suggests that beat and side cooperate in axonal pathway decisions.

\section{Beat regulates the expression levels of Side}

In wild-type embryos, Side is down-regulated shortly after contact with motor axons (e.g., Fig. 1I, asterisk). Since the genetic evidence presented above suggests that Beat is required to detect Side, the distribution of Side might be altered in beat mutants. We analyzed the expression level and subcellular distribution of Side in wild-type and beat mutant embryos employing anti-Side antibodies (Sink et al. 2001). Peripheral nerves normally lack detectable levels of Side at stage 16/17, since Side is efficiently cleared from sensory axons once motor axons have entered their target regions (Fig. 5A). In beat mutants, however, Side was not eliminated, and instead was expressed on sensory axons until late embryonic stages (Fig. 5B). To test whether Beat plays a role in the regulation of Side levels we ectopically expressed Beat either in all post-mitotic neurons or in all muscles in a beat mutant background. Nerve-derived Beat fully rescued the constitutive expression phenotype of Side and induced the elimination of Side from sensory axons (Fig. 5C). Only low traces of Side expression remained (Fig. 5C, arrow). In contrast, muscle-derived Beat failed to down-regulate Side on sensory axons (Fig. 5D). In addition, pan-neuronal (Elav-Gal4) and motor neuronal (FasIIGal4), but not muscular Beat (Mef2-Gal4) expression, rescued the axon guidance phenotype, leading to a neuromuscular innervation pattern almost indistinguishable from wild-type larvae (Table 2). These results suggest that Beat is required cell-autonomously in motor neurons.

Based on the analysis of its primary sequence and its staining pattern "around axons and growth cones" Beat has been suggested to be a secreted protein (Fambrough and Goodman 1996). However, no costaining with an axonal membrane marker has been provided (Fambrough and Goodman 1996). Surprisingly, protein sequence analysis using several topology prediction algorithms (HMMTOP, PredictProtein, TMpred, Phobius, and TopPred) indicates a transmembrane region in Beat (data not shown). We therefore wanted to examine whether Beat is a secreted or membrane-associated protein. In transiently transfected S2 cells, myc-tagged Beat was not 

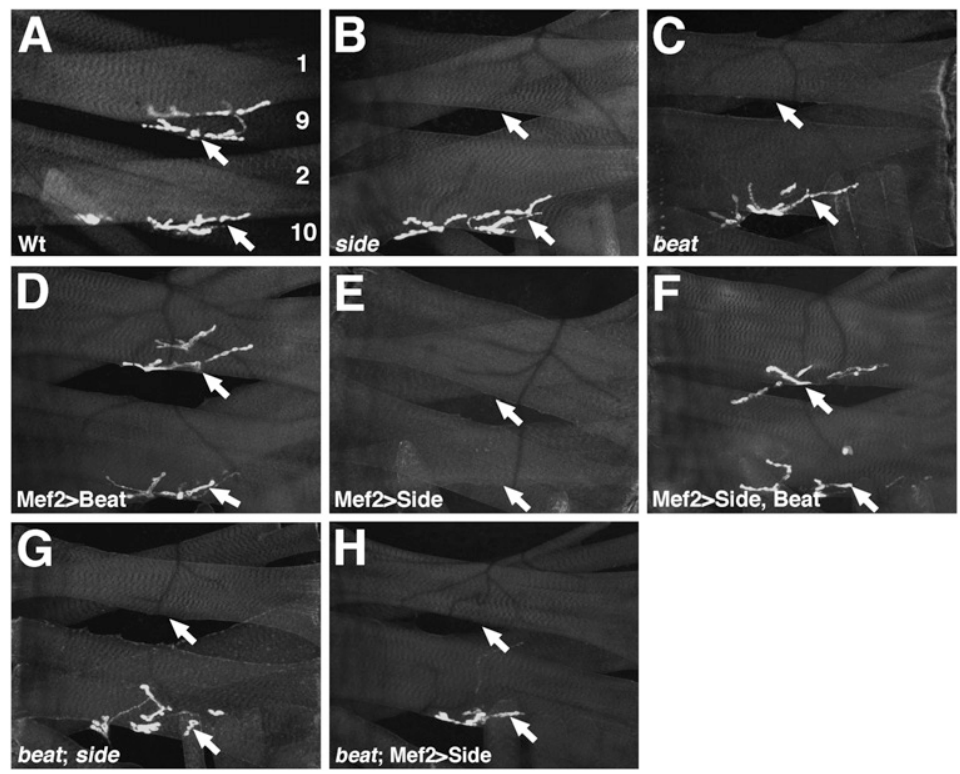

Figure 4. Genetic evidence that Beat and Side function in a common pathway. $(A-H)$ Confocal images of muscle pairs $1 / 9$ and $2 / 10$ in third instar larvae expressing the post-synaptic marker CD8-GFP-Sh. The percentages of noninnervated muscles in the respective genotypes are quantified in Table 2. (A) In wild-type larvae, dorsal-most muscles are innervated by centrally localized NMJs (arrows). $(B, C)$ side $^{C 137} /$ side $^{I 1563}(B)$ and beat $^{3} /$ beat $^{C 163}$ $(C)$ mutant larvae show similar innervation defects on a variety of muscles and frequently lack NMJs on muscles 1/9. (D) Ectopic expression of Beat in muscles of wild-type larvae using Mef2-Gal4 does not affect the innervation of dorsal muscles. (E) Ectopic expression of Side in muscles results in complete lack of NMJs on dorsal-most muscles. $(F)$ Coexpression of Beat and Side in muscles suppresses the innervation defects caused by overexpression of only Side, resulting in a wild-type innervation pattern on dorsal muscles. $(G)$ Phenotypic strength is not increased in beat ${ }^{3} /$ beat $^{\mathrm{C} 163}$; side $e^{\mathrm{C} 137} /$ side $\mathrm{Cl}^{\mathrm{C} 7}$ double mutants when compared with single mutants of beat or side. $(H)$ Overexpression of Side in muscles of beat $^{3} /$ beat $^{\mathrm{C} 163}$ mutant animals does not increase phenotypic strength of beat mutants, leading to the absence of NMJs only on muscles $1 / 9$. secreted into the supernatant (Supplemental Fig. S2). Similar to Side, most of the Beat protein was detected in the organelle fraction but a significant portion was associated with the fraction containing integral membrane proteins (Supplemental Fig. S2). These results combined with the strict cell-autonomous function suggest that Beat functions on cell surfaces.

\section{Beat interacts with Side}

Neuronal Beat might recognize Side-expressing cell surfaces by directly binding to Side or a Side-containing complex. To test if Beat interacts with Side, we transiently transfected S2 cells with tagged constructs of either protein and subjected these cells to aggregation assays. S2 cells expressing either Beat-myc or Side-GFP did not form cell-cell aggregates, indicating that neither Beat nor Side interact homophilically (Fig. 6B,C). In contrast, cells cotransfected with Beat-myc and SideGFP formed large aggregates (Fig. 6D). Large cell clusters also formed when we mixed Beat-myc-expressing cells with Side-GFP-expressing cells (Fig. 6E). We obtained similar results with Beat-Cherry constructs as well (Fig. $6 \mathrm{~F})$. The cell aggregates consisted almost exclusively of

Table 2. Percentage of dorsal and ventral muscles lacking NMJs in third instar larvae of the indicated genotypes expressing CD8GFP-Sh

\begin{tabular}{|c|c|c|c|c|}
\hline Lack of NMJs on dorsal muscles & Muscle 1 & Muscle 9 & Muscle 2 & Muscle 10 \\
\hline Wild type (CD8-GFP-Sh) & 0 & 0 & 0 & 0 \\
\hline beat $^{3} /$ beat ${ }^{\text {C163 }}$ & 31 & 34 & 8 & 13 \\
\hline side ${ }^{C 137}$ side $e^{I 1563}$ & 19 & 24 & 11 & 9 \\
\hline beat $^{3} /$ beat $^{\text {C163 }}$; side sl37 $^{\text {Side }}$ s137 $^{C 137}$ & 24 & 42 & 16 & 20 \\
\hline 24B-Gal4/UAS-side & 88 & 88 & 39 & 39 \\
\hline Mef2-Gal4/UAS-side & 93 & 93 & 60 & 57 \\
\hline UAS-dsRed; Mef2-Gal4/UAS-side & 88 & 86 & 60 & 58 \\
\hline Mef2-Gal4/UAS-beat, UAS-side & 24 & 30 & 14 & 14 \\
\hline Mef2-Gal4/UAS-beat & 16 & 21 & 7 & 5 \\
\hline beat $^{3} /$ beat $^{\text {C163 }}$; 24B-Gal4/UAS-side & 32 & 31 & 10 & 5 \\
\hline beat $^{3} /$ beat $^{\text {C163 }}$; Mef2-Gal4/UAS-side & 25 & 29 & 11 & 9 \\
\hline beat $^{3} /$ beat $^{\text {C163 }}$; Elav-Gal4/UAS-beat & 4 & 2 & 0 & 0 \\
\hline beat $^{3} /$ beat ${ }^{C 163}$; Mef2-Gal4/UAS-beat & 26 & 18 & 8 & 8 \\
\hline FasII-Gal4; beat ${ }^{3} /$ beat $^{\mathrm{C} 163}$; UAS-beat & 5 & 4 & 0 & 1 \\
\hline Lack of NMJs on ventral muscles & Muscle 12 & Muscle 13 & Muscle 6 & Muscle 7 \\
\hline Wild type (CD8-GFP-Sh) & 0 & 0 & 0 & 2 \\
\hline beat $^{3} /$ beat $^{\text {C163 }}$ & 25 & 34 & 38 & 55 \\
\hline $\operatorname{side}^{C 137} /$ side $^{I 1563}$ & 58 & 75 & 77 & 85 \\
\hline beat $^{3} /$ beat $^{C 163} ;$ side $^{C 137} /$ side $^{C 137}$ & 15 & 48 & 72 & 84 \\
\hline
\end{tabular}

$n=100$ hemisegments for each genotype. 


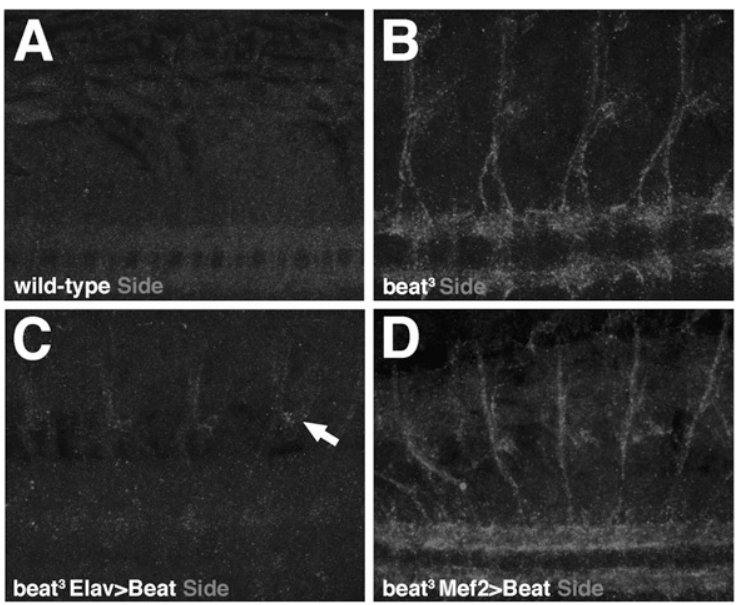

Figure 5. Expression of Beat in neurons but not in muscles regulates the expression level of Side. (A) At stage 16, Side is no longer detected on peripheral nerves of wild-type embryos but remains weakly expressed in muscles and the neuropil. $(B)$ In beat ${ }^{3}$ mutant embryos, Side remains highly expressed on peripheral nerves and the ventral neuropil until the end of embryogenesis. $(C)$ Expression of wild-type Beat in a beat ${ }^{3}$ mutant embryo using Elav-Gal4 rescues the constitutive expression phenotype and induces the down-regulation of Side on peripheral nerves. A few Side-positive particles, however, remain (arrow). (D) Expression of wild-type Beat in muscles of beat $^{3}$ mutant embryos using Mef2-Gal4 fails to down-regulate Side on peripheral nerves, indicating that Beat does act cellautonomously.

Beat- and Side-positive cells, and contained only marginal amounts of nontransfected cells $(<10 \%)$. Consistent with the subcellular fractionation, both Beat and Side were predominantly detected in small granular compartments in the cytoplasm but also at the plasma membrane (Fig. 6F). The formation of large cell clusters indicates that Beat and Side interact heterophilically. To confirm these interactions, we performed immunoprecipitations to isolate protein complexes containing Side-GFP. Beatmyc- and Side-GFP-expressing S2 cells were lysed and incubated with anti-GFP antibodies (Fig. 6G). Beat-myc specifically precipitated with Side-GFP. Nonspecific interaction of Beat-myc with anti-GFP antibodies was not observed (Fig. 6G). In the reverse experiment, Side-myc was specifically precipitated with Beat-GFP-containing protein complexes (Fig. 6H). These results indicate that Beat and Side interact either directly or indirectly to form a heterophilic adhesion complex that, in embryos, might mediate the adhesion between motor axons and the substrate.

\section{Discussion}

In this work, we provided evidence for a simple guidance mechanism that guides Drosophila motor axons to their target regions. During the period of axonal pathfinding, Side is dynamically expressed on different tissues but its temporal shift forms a spatial pattern as such that high levels are located ahead of motor axonal growth cones.
Beat-expressing motor axons recognize and follow Sidelabeled surfaces. In order to better visualize the complex spatial and temporal dynamics of axon guidance processes, we established a FasIIGFP ${ }^{\text {Mue397 }}$-based imaging assay that allowed us to analyze the activity of growth cones in living wild-type and mutant embryos. These
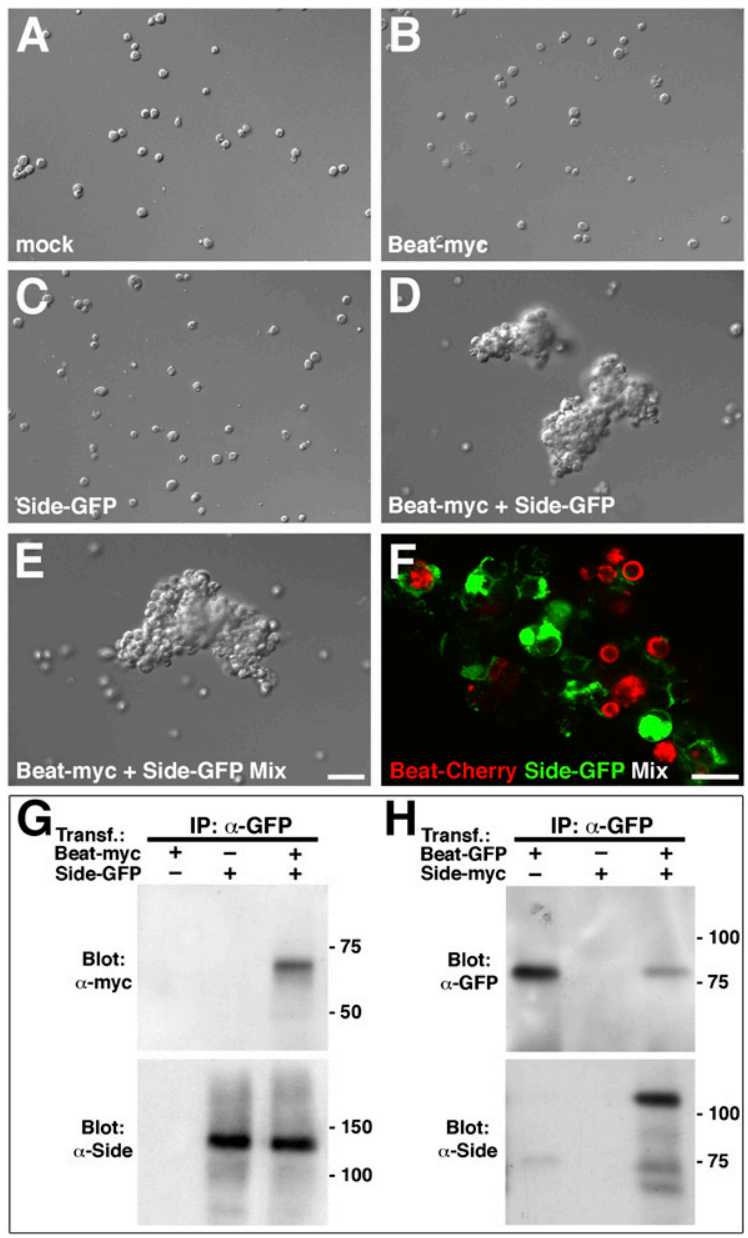

Figure 6. Beat interacts with Side. $(A-F)$ S2 cell aggregation assays. $(A-C)$ Mock transfected $(A)$, Beat-myc transfected $(B)$, or Side-GFP transfected $(C)$ S2 cells do not form homophilic cell aggregates. $(D)$ S2 cells cotransfected with Beat-myc and SideGFP form large cell aggregates. (E) S2 cells transfected with Beatmyc or Side-GFP in discrete culture dishes and mixed together in a common dish form large cell aggregates (Mix experiment). Bar, $30 \mu \mathrm{m}$. $(F)$ S2 cells individually transfected with BeatCherry or Side-GFP were mixed together (Mix experiment). Cell aggregates are comprised of both cell types. Bar, $15 \mu \mathrm{m} .(G, H)$ Immunoprecipitation of protein complexes containing Beat and Side. $(G)$ S2 cells transiently transfected with Beat-myc and/or Side-GFP were immunoprecipitated with anti-GFP antibodies. Immunoblots of the immunoprecipitates (IP) were developed with anti-myc and anti-Side antibodies. Beat-myc is precipitated only in the presence of Side-GFP. $(H)$ S2 cells transiently transfected with Beat-GFP and/or Side-myc were immunoprecipitated with anti-GFP antibodies. Immunoblots of the precipitates were developed with anti-GFP and anti-Side antibodies. Sidemyc is precipitated only in the presence of Beat-GFP. Note that molecular weights depend on the tags used. 
time-lapse observations revealed that in wild-type embryos, the ISN migrates continuously through the lateral body wall until it reaches a choice point near the dorsal trunk. As expected for migration along a substrate pathway, the ISN employs a relatively small growth cone that extends only few filopodia for steering. The observed growth rates are in good agreement with the growth rates of the RP2 axon in filleted, semiviable embryo preparations (Murray et al. 1998).

\section{Side labels the path for motor axons}

The absence of the attractant Side strongly interferes with axonal growth and results in delayed arrival of the ISN in its dorsal target regions, frequently failing to innervate the dorsal-most muscles. The lack of Sidemediated attraction likely prevents the progression at a normal rate, causing the ISN to develop a complex growth cone that appears to actively search for guidance information. Since Side is a transmembrane protein, it is predicted to function as a contact attractant. The spatiotemporal expression pattern should therefore provide pathway information. The growth cone of the ISN follows Side-positive cell surfaces from its first emergence in the ventral nerve cord to its dorsal target region. Consequently, the tight association of motor axons and their substrates-e.g., sensory axons-is partially lost in side mutants. Since Side is expressed in all sensory clusters (Sink et al. 2001), it likely also prefigures the SNa and SNc pathways. Based on the positions of the segmental sensory clusters in the body wall, motor axons following Sidestep-labeled sensory axons are guided into the proximity of their target regions. At stage 15, motor axons reach the end of the sensory tracks and begin to defasciculate into the muscle fields that up-regulate Side at this developmental time point. In this respect, it is interesting to note that motor pathways terminate on the cell bodies of sensory neurons when deprived of their target muscles (Landgraf et al. 1999). The opposed migration of efferent motor axons and afferent sensory axons therefore provides a robust mechanism for the establishment of the basic neuromuscular connectivity pattern in Drosophila. In the brain of vertebrates, a similar mechanism controls the wiring of the thalamus and the cortex (Lopez-Bendito and Molnar 2003). Corticothalamic and thalamocortical fibers meet at a common intermediate target and continue to grow along each other in opposite directions.

During its journey through the lateral body wall, the ISN completely ignores the nearby ventral and lateral muscle fields. At this stage, muscle fibers are not yet differentiated, and hence do not express endogenous Side. If these muscle precursors, however, are forced to express Side prematurely under control of Mef2-Gal4, the ISN diverts from its normal path and grows straight into the muscle field. The premature attraction drastically slows down the migration toward dorsal targets, leading to a permanent lack of NMJs on dorsal muscles. Thus, both gain and loss of Side cause migratory delays that result in the lack of NMJs on dorsal muscles but for different reasons. Ectopic expression of Side leads to excess attrac- tion in wrong directions, whereas lack of Side leads to reduced attraction along the predestined path.

\section{Beat interacts with Side}

Since mutations in both beat and side cause similar axon guidance phenotypes in embryos (Fambrough and Goodman 1996; Sink et al. 2001) and innervation defects in larvae (this study), we assumed that the products of both genes might interact functionally. Several pieces of genetic evidence suggest that beat and side function in a common pathway. First, the double mutant phenotype is similar to the respective single mutant phenotypes. Second, overexpression of Side in muscles of wild-type embryos leads to premature attraction of the ISN into ventral and lateral muscle fields. This gain-of-function phenotype is completely suppressed by coexpression of Beat. Third, overexpression of Side in muscles of beat mutants renders motor axons unresponsive to ectopic Side. Fourth, endogenous Side is not down-regulated in beat mutants. In addition, Beat and Side interact in vitro. S2 cells expressing Beat and Side form large cell clusters in aggregation assays when individually transfected cell populations are mixed. Moreover, Beat coimmunoprecipitates with Side, supporting the idea that Beat interacts with Side or a Side-containing complex. The formation of cell aggregates further argues that Beat-Side interactions lead to the formation of heterophilic adhesion complexes.

The spatiotemporal expression of Side appears to be strictly regulated. The levels of Side expression are highest in front of motor axonal growth cones. Side disappears from cell surfaces once these cells have been contacted by motor axons, indicating that motor nerves neutralize attractive surfaces and thereby disguise the path they are following. Motor nerve bundles that exit the CNS at a later time point are thus prevented from choosing the same route. In beat mutants, this regulatory mechanism appears to be nonfunctional. Side is constitutively expressed in peripheral nerves. Expression of exogenous Beat in post-mitotic neurons but not in muscles rescued the regulatory defects, suggesting that Beat induces the down-regulation of Side cell-autonomously. If Beat would be a secreted protein, one would expect that expression in muscles down-regulates Side on sensory axons. The secreted metalloprotease tolloid-related (Meyer and Aberle 2006; Serpe and O'Connor 2006) as well as the secreted TGF- $\beta$ ligand Dawdle (Parker et al. 2006) have been shown to rescue axonal guidance defects tissue independently. Biochemical data from transiently transfected S2 cells further support a cell-autonomous function for Beat. Beat was not secreted into the medium, and a fraction of it was associated with membranes. However, since Beat is not normally expressed in S2 cells the lack of a coreceptor or chaperone might prevent its secretion. Candidate coreceptors are the remaining members of the beat multigene family (Pipes et al. 2001). Several family members are expressed in the ventral nerve cord, and might function together with Beat in the regulation of its subcellular localization and/or function (Pipes et al. 2001). Further experiments will be necessary to determine the 
composition of Beat-containing complexes, and how they transduce guidance signals into the growth cone.

\section{Beat-Side complexes determine the growth direction of motor axons}

If Side is an instructive signal for Beat-expressing motor axons it should be possible to redirect their paths in a side mutant background; i.e., in the absence of endogenous Side but in the presence of exogenous Side on a defined tissue. Regardless of whether Side is expressed in trachea, muscles, or hemocytes in a side mutant background, motor axons head toward the ectopic source of Side. The growth cones find and recognize Side-expressing cell surfaces and adopt their route accordingly. In the most extreme case, motor axons strongly interacted with hemocytes, which are highly motile cells. Side therefore potently controls the path of motor axons. Although Sidemediated attraction is likely not the only mechanism to reach the muscle targets, these results, together with the high penetrance of sidestep mutant phenotypes, suggest that it is one of the major mechanisms. In this respect, it is important to note that Side is not required for motor axon outgrowth per se, but rather for the specification of the growth direction. Based on the experimental evidence, we propose a model for the navigation of motor axons from the ventral nerve cord to their target area in Drosophila (Fig. 7). Beat-expressing motor axon fascicles recognize, extend on, and subsequently mask a pre-
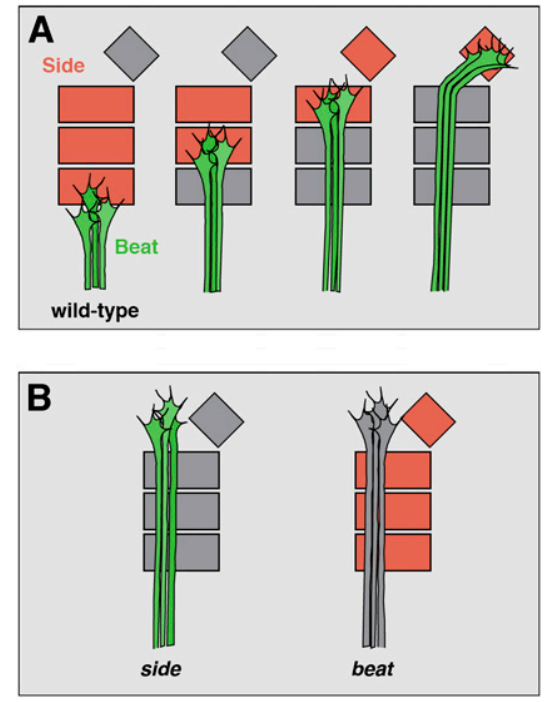

Figure 7. Model: Beat-expressing motor axons follow a Sidelabeled substrate pathway. (A) In wild-type embryos, Beatexpressing motor axons (green) recognize and follow Sidelabeled cell surfaces (red). Contact with motor axons induces the down-regulation of Side (gray). Growth cones migrate until the end of the Side expression domain. Developmentally controlled up-regulation of Side in another tissue induces growth cone turning. $(B, l e f t)$ In side mutants, growth cones fail to turn, as substrates are not labeled. Other possible phenotypes such as delays or detours are not depicted. (Right) In beat mutants, the Side-labeled pathway is constitutively marked but cannot be recognized, thereby preventing growth cone turning. existing, Side-labeled substrate pathway that determines their growth direction (Fig. 7A). In the absence of the labeled pathway, in side mutants, axonal migration is delayed or growth cones head into aberrant directions. In either case they will miss their targets (Fig. 7B). In beat mutants, the pathway is constitutively labeled but cannot be recognized, leading to similar phenotypes (Fig. 7B). Since Beat and Side are conserved in insects, similar guidance principles might occur in all organisms, in which the peripheral nervous system develops from sensory organ precursors.

\section{Materials and methods}

\section{Genetics and fly stocks}

The exon trap line Mue397 was identified in the Muenster 2004 exon trap screen performed by the DFG Consortium Cell Polarity (SPP1111) (Rasse et al. 2005; Stork et al. 2008). The side $^{\mathrm{C} 137}$ and side $\mathrm{I1563}^{\mathrm{I}}$ alleles were isolated in an EMS mutagenesis screen for recessive mutations affecting the structure of NMJs (Aberle et al. 2002). beat ${ }^{3}$, beat ${ }^{\mathrm{C} 163}$, and UAS-dsRED were obtained from the Bloomington stock center. UAS-side ${ }^{29 A}$ and UAS-beat ${ }^{5}$ were kindly provided by C.S. Goodman. The following Gal4-lines were used: 24B-Gal4, Mef2-Gal4 (gifts of C.S. Goodman), breathless (btlGal4; gift of M.A. Krasnow), SerpentGal4 (gift of R. Reuter), FasII-Gal4 (Mz507; B. Altenhein, pers. comm.). As wild-type control strains, $\mathrm{y} \mathrm{w}^{1}$ or $\mathrm{w}^{1}$; CD8-GFP-Sh (Zito et al. 1999) were used.

\section{Molecular biology}

Full-length beat Ia and side cDNAs (kindly provided by C.S. Goodman) were amplified by PCR and cloned into Gateway Entry Vectors (pENTR D-TOPO, Invitrogen). The inserts were sequenced and subcloned into pTWH, pTWM, and pTWG Destination Vectors (Drosophila Genomics Resource Center; donated by Terence Murphy) by LR in vitro recombination, containing UAST promotors and C-terminal 3xHA, 6xMyc, and eGFP tags. The pTWC vector encoding a C-terminal Cherry tag was a generous gift of $\mathrm{F}$. Rodrigues and C. Klämbt. The pUAST vector encoding Slit-myc was kindly provided by J. Hillebrand and C. Klämbt, and the Actin5C-Gal4 vector was provided by A. Wodarz. All steps were performed according to the manual of the manufacturer (Escherichia coli Expression System with Gateway Technology, Invitrogen). Sequence analysis and topology prediction (HMMTOP, PredictProtein, TMpred, and TopPred) were performed at the ExPASy and EBI Proteomics servers.

\section{Immunohistochemistry}

For immunohistochemical stainings, embryos were dechorionated, fixed with $3.7 \%$ formaldehyde and devitellinized. Embryos were washed with PTx (PBS containing 0.1\% Triton X-100) and blocked in PTx $/ 5 \%$ normal goat serum. Primary antibodies were added overnight at $4^{\circ} \mathrm{C}$. Stainings were developed with fluorescently labeled secondary antibodies. The dilutions of the primary antibodies were as follows: mouse anti-Fasciclin II (1D4) 1:20, mouse anti-Sidestep (9B8) 1:20, and mouse anti-Repo (8D12) 1:40 (all gifts of C.S. Goodman), mouse anti-myc (9E10) 1:10 and mouse anti-Futsch (22C10) 1:100 (both from Developmental Studies Hybridoma Bank), mouse anti-GFP 1:400 (Roche), and rabbit anti-GFP 1:1000 (Torrey Pines Biolabs). Cy3- and Alexa488-conjugated secondary antibodies were 
diluted 1:400 (Jackson Immunoresearch; Molecular Probes). The Rhodamine-conjugated chitin-binding probe (New England Biolabs) was diluted 1:400 and consists of the maltose-binding protein fused to a C-terminal region of chitinase A1. Stained embryos were imaged as whole mounts using a LSM510 confocal laser scanning microscope (Zeiss). Projections and single images were adjusted for brightness, contrast, and color matching using linear functions of Adobe Photoshop.

\section{Cell aggregation assays and biochemical methods}

S2 cells were grown in complete Schneider medium supplemented with $10 \%$ fetal calf serum and $50 \mathrm{U} / \mathrm{mL}$ Penicillin/ Streptomycin. Cells $\left(3 \times 10^{6}\right)$ were seeded into six-well plates and transiently transfected using calcium phosphate precipitation (5 $\mu \mathrm{g}$ per vector). The medium was replaced after $16 \mathrm{~h}$. For cell aggregation assays, cells were separated by pipetting and seeded into uncoated $6-\mathrm{cm}$ plastic dishes $3 \mathrm{~d}$ post-transfection. Cells were agitated on a rotary shaker $(100 \mathrm{rpm})$ for $2 \mathrm{~h}$. Aggregates were transferred onto microscope slides and imaged using a Zeiss AxioPlan-2 microscope adjusted for DIC. For the detection of secreted proteins, transfected S2 cells (3-d-old cultures) were incubated for $24 \mathrm{~h}$ in serum-free medium. Cells were pelleted, washed and lysed in $2 \times$ SDS sample buffer. The medium was mixed with an equal volume of $50 \%(\mathrm{w} / \mathrm{v})$ TCA (trichloroacetic acid) (Sigma) and incubated for $30 \mathrm{~min}$ on ice. Precipitated proteins were collected $\left(14,000 \mathrm{rpm}, 30 \mathrm{~min}, 4^{\circ} \mathrm{C}\right)$ and washed with ice-cold isopropanol. The pellet was solubilized in $2 \times$ SDS sample buffer and titrated with $1 \mathrm{M}$ Tris ( $\mathrm{pH} 7.5)$. For subcellular fractionation, S2 cells were lysed by sonification and subjected to differential centrifugation $(5000 \mathrm{rpm}, 24,000 \mathrm{rpm}$, and $75,000 \mathrm{rpm}$ for $30 \mathrm{~min}$ at $4^{\circ} \mathrm{C}$ in an ultracentrifuge [Optima Max-E, Beckman Coulter]). The pellet was incubated with 0.1 M $\mathrm{Na}_{2} \mathrm{CO}_{3}$ (pH 11.5) to release peripheral membrane proteins.

\section{Immunoprecipitation and immunoblotting}

S2 cells from aggregation assays were collected by centrifugation and lysed in lysis buffer (50 mM Tris at $\mathrm{pH} 7.5,150 \mathrm{mM} \mathrm{NaCl}$, $1 \%$ NP40, protease inhibitor cocktail [Roche]). Insoluble debris was pelleted by centrifugation ( $\left.15 \mathrm{~min}, 14,000 \mathrm{rpm}, 4^{\circ} \mathrm{C}\right)$. The supernatants were incubated with $1 \mu \mathrm{g}$ of rabbit anti-GFP antibodies (Invitrogen) for $1 \mathrm{~h}$ at room temperature on a rotary shaker. Protein complexes were collected by addition of equilibrated protein A sepharose beads and washed with washing buffer (50 mM Tris at $\mathrm{pH} 7.5,150 \mathrm{mM} \mathrm{NaCl}, 1 \% \mathrm{NP} 40)$. Bound proteins were eluted by boiling in $2 \times$ SDS sample buffer. Samples were separated on $7.5 \%$ SDS-PAGE gels (Bio-Rad) and transferred onto PVDF membranes (GE Healthcare) by semidry blotting (Biozym). Blots were incubated with anti-Side antibodies (9B8) (1:10) or anti-myc antibodies (9E10) (1:100; Developmental Studies Hybridoma Bank) and developed with HRP-conjugated secondary antibodies (1:10000; Dianova) followed by enhanced chemiluminescence detection (ECL, GE Healthcare). Molecular weights were standardized with Precision Plus Protein Dual Color Standards (Bio-Rad).

\section{Time-lapse imaging}

Living, GFP-expressing embryos were dechorionated, mounted in $70 \%$ glycerol/PBS, and immediately examined for still images. For time-lapse movies, embryos were dechorionated, placed on a coverslip, and gently covered with a piece of fruit agar $(0.8 \times$ $0.8 \times 0.2 \mathrm{~cm})$. Liquid agarose $\left(0.4 \%, 42^{\circ} \mathrm{C}\right)$ was then pipetted between the coverslip and the fruit agar block to embed the embryos. The assembly (coverslip up, agar block down) was placed onto a greased glass ring $(0.4 \mathrm{~cm}$ high, $1 \mathrm{~cm}$ diameter). The glass ring was fixed before on a microscope slide with vacuum grease and contained a drop of liquid agarose to support the agar block after solidifying. Time-lapse imaging was performed at an upright confocal laser scanning microscope LSM510 Meta (Zeiss) using a $40 \times$ objective. Stacks of four to five Z-planes were acquired in 45-sec intervals for up to $5 \mathrm{~h}$. Stacks were processed and exported as Quicktime movies using LSM software. Growth cone migration was quantified by measuring the advance of its center in time.

\section{Acknowledgments}

We are indebted to Christian Klämbt for providing laboratory space and support. We thank Stephan Sigrist for recommending the line Mue397 for live imaging, and Benjamin Altenhein for the FasII-Gal4 (Mz507) line. Further fly stocks and reagents have been generously provided by Corey Goodman, Mark Krasnow, Rolf Reuter, Floriano Rodrigues, Jens Hillebrand, Sven Bogdan, Andreas Wodarz, and the Bloomington Stock Center. We are grateful to Christian Klämbt, Sven Bogdan, Thomas Hummel, and Joanne Yew for critical comments on the manuscript. This project was funded by the Deutsche Forschungsgemeinschaft (AB116/3-1, AB116/4-1).

\section{References}

Aberle, H., Haghighi, A.P., Fetter, R.D., McCabe, B.D., Magalhaes, T.R., and Goodman, C.S. 2002. wishful thinking encodes a BMP type II receptor that regulates synaptic growth in Drosophila. Neuron 33: 545-558.

Araujo, S.J. and Tear, G. 2003. Axon guidance mechanisms and molecules: Lessons from invertebrates. Nat. Rev. Neurosci. 4: 910-922.

Bate, C.M. 1976. Pioneer neurones in an insect embryo. Nature 260: $54-56$.

Bazan, J.F. and Goodman, C.S. 1997. Modular structure of the Drosophila Beat protein. Curr. Biol. 7: R338-R339. doi: 10.1016/S0960-9822(06)00168-0.

Buszczak, M., Paterno, S., Lighthouse, D., Bachman, J., Planck, J., Owen, S., Skora, A.D., Nystul, T.G., Ohlstein, B., Allen, A., et al. 2007. The carnegie protein trap library: A versatile tool for Drosophila developmental studies. Genetics 175: 1505-1531.

de Jong, S., Cavallo, J.A., Rios, C.D., Dworak, H.A., and Sink, H. 2005. Target recognition and synaptogenesis by motor axons: Responses to the sidestep protein. Int. J. Dev. Neurosci. 23: 397-410.

Dickson, B.J. 2002. Molecular mechanisms of axon guidance. Science 298: 1959-1964.

Fambrough, D. and Goodman, C.S. 1996. The Drosophila beaten path gene encodes a novel secreted protein that regulates defasciculation at motor axon choice points. Cell 87: 10491058.

Ghysen, A. 1978. Sensory neurones recognise defined pathways in Drosophila central nervous system. Nature 274: 864-872.

Ghysen, A. and Janson, R. 1980. Sensory pathways in Drosophila central nervous system. In Development and neurobiology of Drosophila (eds. O. Siddiqi et al.), pp. 247-265. Plenum Publishing Corp., New York.

Goodman, C.S., Raper, J.A., Chang, S., and Ho, R. 1983. Grasshopper growth cones: Divergent choices and labeled pathways. Prog. Brain Res. 58: 283-304.

Grenningloh, G., Rehm, E.J., and Goodman, C.S. 1991. Genetic analysis of growth cone guidance in Drosophila: Fasciclin II functions as a neuronal recognition molecule. Cell 67: 45-57. 
Holmes, A.L. and Heilig, J.S. 1999. Fasciclin II and Beaten path modulate intercellular adhesion in Drosophila larval visual organ development. Development 126: 261272.

Huber, A.B., Kolodkin, A.L., Ginty, D.D., and Cloutier, J.F. 2003. Signaling at the growth cone: Ligand-receptor complexes and the control of axon growth and guidance. Annu. Rev. Neurosci. 26: 509-563.

Hummel, T., Krukkert, K., Roos, J., Davis, G., and Klambt, C. 2000. Drosophila Futsch/22C10 is a MAP1B-like protein required for dendritic and axonal development. Neuron 26: 357-370.

Katz, M.J. and Lasek, R.J. 1979. Substrate pathways which guide growing axons in Xenopus embryos. J. Comp. Neurol. 183: $817-831$.

Katz, M.J. and Lasek, R.J. 1981. Substrate pathways demonstrated by transplanted Mauthner axons. J. Comp. Neurol. 195: 627-641.

Landgraf, M., Baylies, M., and Bate, M. 1999. Muscle founder cells regulate defasciculation and targeting of motor axons in the Drosophila embryo. Curr. Biol. 9: 589-592.

Lopez-Bendito, G. and Molnar, Z. 2003. Thalamocortical development: How are we going to get there? Nat. Rev. Neurosci. 4: 276-289.

Meyer, F. and Aberle, H. 2006. At the next stop sign turn right: The metalloprotease Tolloid-related 1 controls defasciculation of motor axons in Drosophila. Development 133: 40354044.

Murray, M.J., Merritt, D.J., Brand, A.H., and Whitington, P.M. 1998. In vivo dynamics of axon pathfinding in the Drosophilia CNS: A time-lapse study of an identified motorneuron. J. Neurobiol. 37: 607-621.

Mushegian, A.R. 1997. The Drosophila Beat protein is related to adhesion proteins that contain immunoglobulin domains. Curr. Biol. 7: R336-R338. doi: 10.1016/S0960-9822(06)00167-9.

Parker, L., Ellis, J.E., Nguyen, M.Q., and Arora, K. 2006. The divergent TGF- $\beta$ ligand Dawdle utilizes an activin pathway to influence axon guidance in Drosophila. Development 133: 4981-4991.

Pipes, G.C., Lin, Q., Riley, S.E., and Goodman, C.S. 2001. The Beat generation: A multigene family encoding IgSF proteins related to the Beat axon guidance molecule in Drosophila. Development 128: 4545-4552.

Raper, J.A., Bastiani, M., and Goodman, C.S. 1983a. Pathfinding by neuronal growth cones in grasshopper embryos. II. Selective fasciculation onto specific axonal pathways. J. Neurosci. 3: $31-41$

Raper, J.A., Bastiani, M.J., and Goodman, C.S. 1983b. Guidance of neuronal growth cones: Selective fasciculation in the grasshopper embryo. Cold Spring Harb. Symp. Quant. Biol. 48: $587-598$

Rasse, T.M., Fouquet, W., Schmid, A., Kittel, R.J., Mertel, S., Sigrist, C.B., Schmidt, M., Guzman, A., Merino, C., Qin, G., et al. 2005. Glutamate receptor dynamics organizing synapse formation in vivo. Nat. Neurosci. 8: 898-905.

Serpe, M. and O'Connor, M.B. 2006. The metalloprotease tolloid-related and its TGF- $\beta$-like substrate Dawdle regulate Drosophila motoneuron axon guidance. Development 133: 4969-4979.

Silver, J. and Rutishauser, U. 1984. Guidance of optic axons in vivo by a preformed adhesive pathway on neuroepithelial endfeet. Dev. Biol. 106: 485-499.

Singer, M., Nordlander, R.H., and Egar, M. 1979. Axonal guidance during embryogenesis and regeneration in the spinal cord of the newt: The blueprint hypothesis of neuronal pathway patterning. J. Comp. Neurol. 185: 1-21.
Sink, H. and Whitington, P.M. 1991. Location and connectivity of abdominal motoneurons in the embryo and larva of Drosophila melanogaster. J. Neurobiol. 22: 298-311.

Sink, H., Rehm, E.J., Richstone, L., Bulls, Y.M., and Goodman, C.S. 2001. sidestep encodes a target-derived attractant essential for motor axon guidance in Drosophila. Cell 105: 57-67.

Sperry, R.W. 1963. Chemoaffinity in the orderly growth of nerve fiber patterns and connections. Proc. Natl. Acad. Sci. 50: 703-710.

Stork, T., Engelen, D., Krudewig, A., Silies, M., Bainton, R.J., and Klambt, C. 2008. Organization and function of the bloodbrain barrier in Drosophila. J. Neurosci. 28: 587-597.

van Vactor, D., Sink, H., Fambrough, D., Tsoo, R., and Goodman, C.S. 1993. Genes that control neuromuscular specificity in Drosophila. Cell 73: 1137-1153.

Zito, K., Parnas, D., Fetter, R.D., Isacoff, E.Y., and Goodman, C.S. 1999. Watching a synapse grow: Noninvasive confocal imaging of synaptic growth in Drosophila. Neuron 22: 719729. 


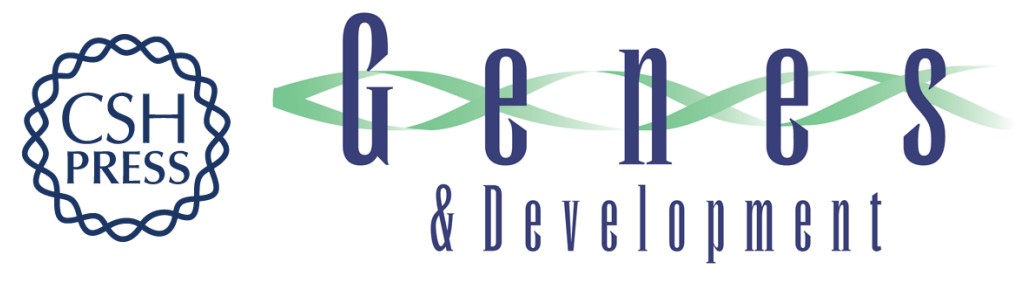

\section{Drosophila motor axons recognize and follow a Sidestep-labeled substrate pathway to reach their target fields}

Matthias Siebert, Daniel Banovic, Bernd Goellner, et al.

Genes Dev. 2009, 23: originally published online April 15, 2009

Access the most recent version at doi:10.1101/gad.520509

\section{Supplemental http://genesdev.cshlp.org/content/suppl/2009/04/16/gad.520509.DC1 \\ Material}

Related Content Choosing the road less traveled by: a ligandreceptor system that controls target recognition by Drosophila motor axons

Kai Zinn

Genes Dev. May , 2009 23: 1042-1045

References This article cites 35 articles, 11 of which can be accessed free at: http://genesdev.cshlp.org/content/23/9/1052.full.html\#ref-list-1

Articles cited in:

http://genesdev.cshlp.org/content/23/9/1052.full.html\#related-urls

\section{License}

Email Alerting

Service

Receive free email alerts when new articles cite this article - sign up in the box at the top right corner of the article or click here.

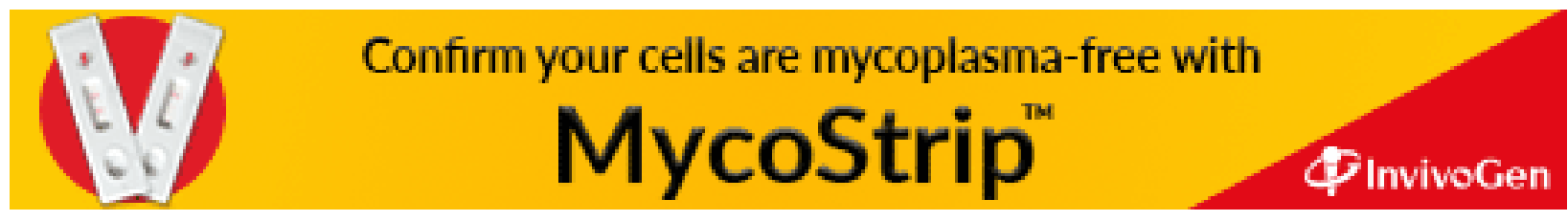

